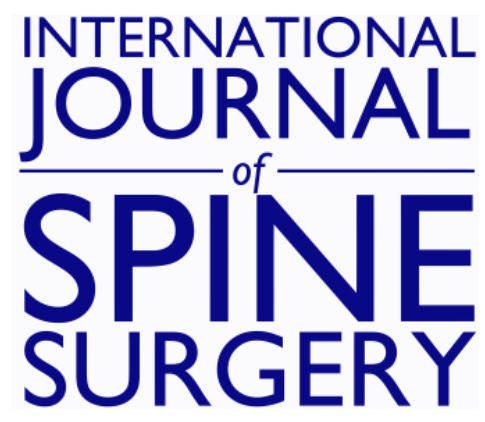

\title{
The Efficacy of Liposomal Bupivacaine in Lumbar Spine Surgery
}

LUKE BROWN, TRISTAN WEIR, MARK SHASTI, OMER YOUSAF, IMRAN YOUSAF, OLIVER TANNOUS, EUGENE KOH, KELLEY BANAGAN, DANIEL GELB and STEVEN LUDWIG

Int J Spine Surg 2018, 12 (4) 434-440

doi: https://doi.org/10.14444/5052

http://ijssurgery.com/content/12/4/434

This information is current as of April 25, 2023.

Email Alerts Receive free email-alerts when new articles cite this article. Sign up at: http://ijssurgery.com/alerts 


\title{
The Efficacy of Liposomal Bupivacaine in Lumbar Spine Surgery
}

\author{
LUKE BROWN, MD, MBA, TRISTAN WEIR, MD, MARK SHASTI, MD, OMER YOUSAF, MD, IMRAN \\ YOUSAF, DO, OLIVER TANNOUS, MD, EUGENE KOH, MD, PHD, KELLEY BANAGAN, MD, DANIEL \\ GELB, MD, STEVEN LUDWIG, MD \\ Department of Orthopaedics, University of Maryland Medical Center, University of Maryland, Baltimore, MD
}

\begin{abstract}
Background: Postoperative pain management in spine surgery holds unique challenges. The purpose of this study is to determine if the local anesthetic liposomal bupivacaine (LB) reduces the total opioid requirement in the first 3 days following posterior lumbar decompression and fusion (PLDF) surgery for degenerative spondylosis.

Methods: Fifty patients underwent PLDF surgery in a prospective randomized control pilot trial between August 2015 and October 2016 and were equally allocated to either a treatment (LB) or a control (saline) group. Assessments included the 72-hour postoperative opioid requirement normalized to 1 morphine milligram equivalent (MME), visual analog scale (VAS), and hospital length of stay.

Results: LB did not significantly alter the 72-hour postoperative opioid requirement compared to saline (11.6 vs. 13.4 MME, $P=.40$ ). In a subgroup analysis, there was also no significant difference in opioid consumption among narcotic-naive patients with either LB or saline. Among narcotic tolerant patients, however, opioid consumption was higher with saline than LB (20.6 MME vs. 13.3 MME, $P=.048)$. Additionally, pre- and postoperative VAS scores and hospital length of stay were not significantly different with either LB or saline.

Conclusions: In the setting of PLDF surgery, LB injections did not significantly reduce the consumption of opioids in the first 3 postoperative days, nor did the hospital length of stay or VAS pain scores, compared to saline. However, LB could be beneficial in reducing the consumption of opioids in narcotic-tolerant populations.
\end{abstract}

Level of Evidence: 2.

Lumbar Spine

Keywords: degenerative spondylosis, liposomal bupivacaine, lumbar decompression and fusion, opioid, postoperative pain

\section{INTRODUCTION}

Posterior lumbar spinal decompression and fusion procedures are common treatment modalities for patients suffering from degenerative spondylosis and/or lumbar spinal stenosis. ${ }^{1-3}$ These procedures often require paraspinal muscle dissection, bone resection for the laminectomy, decortication for fusion bed preparation, and manipulation of vertebrae with instrumentation. Achieving adequate postoperative pain control in these patients is therefore critical and often involves a multimodal approach. ${ }^{4}$ Current regimens involve the use of opioid, nonopioid analgesics, and/or local anesthetics. $^{5-7}$ Local infiltration of anesthetics in the surgical site during wound closure is commonly performed with lumbar spine surgery. 8,9 These agents are traditionally used to provide postoperative analgesia, but the effects are limited by dosing restrictions due to potential systemic side effects from peak plasma concentrations. ${ }^{10}$ Furthermore, the duration of analgesia is limited by each anesthetic's halflife. ${ }^{11}$ Postoperative pain relief is frequently managed with oral and/or intravenous opioids. However, the significant use of opioids may lead to wellestablished opioid-related adverse events, such as nausea, vomiting, constipation, urinary retention, and respiratory depression. ${ }^{12-14}$ These complications can protract hospitalizations, promote patient dissatisfaction, and rapidly expend health care resources. ${ }^{15}$ In an effort to reduce these burdens and complications, a novel nonopioid was developed to block transmission of pain from nociceptive nerve endings. ${ }^{16}$

Liposomal bupivacaine (LB; Pacira Pharmaceuticals, Parsippany, New Jersey) is a long-acting injectable local anesthetic that can provide postoperative analgesia for up to 72 hours. ${ }^{11}$ Its longer 
duration of action and lower risk of systemic absorption is due to its formulation, which consists of bupivacaine loaded into multivesicular liposomes using a proprietary Depofoam technology. The components consist of common phospholipids, cholesterol, and triglycerides with over $97 \%$ consisting of water and 3\% lipids, helping to arrange the liposome in a honeycomb configuration. ${ }^{29}$ This preparation allows for a slower release of bupivacaine from the liposome, resulting in a delay in peak plasma concentrations. ${ }^{11}$ The highest Food and Drug Administration-approved dose (266 mg) used in this study has been shown to have a peak plasma concentration of 24 hours (0.5-49 hours) and a mean half-life of $17.1 \pm 6.8$ hours following its use in total knee arthroplasty. ${ }^{11}$

The safety and efficacy profile of LB has been well established in nonorthopedic procedures, including hemorrhoidectomy, bunionectomy, and breast augmentation procedures. ${ }^{15-18}$ However, its role in managing postoperative pain in patients undergoing spine surgery is not well understood. Due to the paucity of literature on this topic, the primary goal of this study is to determine the efficacy of LB following posterior lumbar decompression and fusion (PLDF) in a prospective, randomized placebo-controlled trial. The study hypothesized no significant change in the total inpatient use of opioids during the first 72 hours after surgery, including pre- and postoperative visual analog scale (VAS) pain scores, and hospital length of stay.

\section{METHODS}

\section{Study Design}

This study was funded by Pacira Pharmaceuticals Inc. with no conflict of interest. After obtaining institutional review board approval, 50 patients were enrolled in a prospective randomized control trial from August 2015 to October 2016. Patients 18 years of age or older undergoing a primary elective, open posterior lumbar decompression and instrumented fusion surgery ( 1 or 2 level) for degenerative spondylosis were included. Patients with an active infection, primary or metastatic malignancy of the spine, fracture of a lumbar vertebra, history of narcotic or other substance abuse, impaired cardiovascular function, or severe hepatic disease were excluded. All procedures were performed by 4 fellowship-trained orthopaedic spine surgeons at a tertiary referral center and included decompression plus or minus medial facetectomies and foraminotomies as well as posterior instrumentation with pedicle screws and rod constructs. Fusion techniques included posterolateral, posterior lumbar interbody, and transforminal interbody fusions with local bone autograft, morcelized allograft bone, and/or demineralized bone matrix in any combination.

Patients were randomized in a 1:1 fashion by our hospital research pharmacy to ensure that they were equally allocated to either a treatment or a control group. Computer randomization was used to assign patients to their respective groups. Depending on the patient's random assignment, the research pharmacy staff delivered either LB or the placebocontrol medication to the operating room. The treatment group received $266 \mathrm{mg}$ of $\mathrm{LB}$ in a $60-\mathrm{mL}$ suspension, while the control group received $60 \mathrm{~mL}$ of $0.9 \%$ sterile saline. Local injections were made prior to wound closure into the exposed paraspinal muscles and surrounding soft tissues. Due to the noticeable appearance of LB suspension compared to placebo saline, research staff and investigators administering the injections were not blinded to patient group assignments. All other providers involved in postoperative care-including nurses, pain management team members, and physical therapists - were blinded to patient group assignments. All patients had a patient-controlled analgesia (PCA) pump initiated shortly after surgery that was discontinued at 6:00 AM on postoperative day 1 . Intravenous (IV) and oral (PO) opioid-based pain medications were used only as necessary for adequate pain control throughout the postoperative period in conjunction with other nonopioid medications.

\section{Data Collection and Statistical Analysis}

Cumulative data were entered into an electronic data sheet (Microsoft Excel, Microsoft Office, Redmond, Washington). Demographic data included age, sex, body mass index (BMI), and smoking status. Surgical data included operation length, estimated blood loss, and number of vertebrae that were decompressed and fused. For further subgroup analysis, patients were identified to be either narcotic tolerant (NT) or narcotic naive $(\mathrm{NN})$. Patients taking opioids before the time of the surgery were considered to be NT. Patients not 
Table 1. Morphine milligram equivalent (MME) conversion table as by Grieff et al. $^{25}$

\begin{tabular}{lc}
\hline Opioid (Route) & $\begin{array}{c}\text { Equivalency Factor to } \\
\text { 10 mg IV Morphine (1 MME) }\end{array}$ \\
\hline Morphine (IV) & $10 \mathrm{mg}$ \\
Morphine (PO) & $30 \mathrm{mg}$ \\
Hydromorphone (IV) & $1.5 \mathrm{mg}$ \\
Hydromorphone (PO) & $7.5 \mathrm{mg}$ \\
Oxycodone (PO) & $20 \mathrm{mg}$ \\
OxyContin (PO) & $20 \mathrm{mg}$ \\
Hydrocodone (PO) & $30 \mathrm{mg}$ \\
Codeine (PO) & $200 \mathrm{mg}$ \\
Tramadol (PO) & $500 \mathrm{mg}$ \\
Fentanyl (IV) & $0.2 \mathrm{mg}$ \\
Meperidine (IV) & $75 \mathrm{mg}$ \\
\hline
\end{tabular}

Abbreviations: IV, intravenous; PO, oral.

${ }^{1}$ As per our institution.

taking any opioids at the time of surgery were considered to be NN.

The primary outcome measure was the total 72hour postoperative opioid consumption between the treatment and control group. At the end of the 72 hour postoperative period, the dosages $(\mathrm{mg})$ for all PCA, IV, and PO opioid analgesics consumed by each patient were normalized to $10 \mathrm{mg}$ of IV morphine, or 1 morphine milligram equivalent (MME), as done by Grieff et al (Table 1). ${ }^{28}$ Mean PCA, IV, and PO MME values were then calculated for each group. To calculate the total opioid consumption, the mean PCA, IV, and PO MME values were added. Secondary outcome measures included pain scores and hospital length of stay. To assess pain scores, a VAS survey was administered preoperatively on the day of surgery as well as the mornings of postoperative days 1 through 3. Data regarding hospital length of stay were collected from the day of admission until the patient was successfully discharged.

Statistical analysis was done with the aid of JMP Pro Version 13 software (SAS Institute Inc., Cary, North Carolina). Statistical tests performed included a 2-tailed Fischer exact test for proportional differences in dichotomous variables and a 2-tailed Student $t$ test for the means of normally distributed continuous variables. Nonparametric data were compared using the Wilcoxon rank sum test. An alpha error of $5 \%$ was set as the threshold for significance for all tests.

\section{RESULTS Baseline Characteristics}

Fifty patients were enrolled, with 24 assigned to the treatment group and 26 assigned to the control group. Both groups were similar with regard to age, sex, BMI, Charleston Comorbidity Index scores, ${ }^{30}$ and smoking status (Table 2). Surgical data, including operation length, estimated blood loss, and the number of decompressed/fused vertebrae, were also similar between the treatment and control groups (Table 3).

\section{Postoperative Opioid Consumption}

Data analysis revealed no significant difference in total opioid consumption between the treatment and control group during the first 72 hours postoperatively (11.6 vs. $13.4 \mathrm{MME}, P=.40$ ). When analyzing opioid consumption through different routes of administration (PCA vs. IV rescue vs. PO), no difference was detected between either of the groups, as demonstrated in Table 4. NT and NN patients were further isolated for a subgroup analysis (Table 5). NT patients required a significantly greater total amount of opioids than those who were $\mathrm{NN}$ (16.5 vs. 9.9 MME, $P=.030)$ regardless of the study group. In comparing NT patients of both the treatment and the control group, NT patients in the treatment group required significantly less postoperative opioids (13.3 vs. 20.6 MME, $P=.048)$. Further analysis showed no significant difference in opioid consumption among NN patients between the treatment and control groups (10.2 vs. 9.6 MME, $P=.72$ ).

\section{VAS Pain Scores and Hospital Length of Stay}

There was no significant difference in VAS pain scores between the treatment and control groups

Table 2. Patient characteristics. ${ }^{1}$

\begin{tabular}{|c|c|c|c|}
\hline Variable & Liposomal Bupivacaine Group $(\mathrm{N}=\mathbf{2 4})$ & Control Group $(\mathrm{N}=26)$ & $P$ Value \\
\hline Male:female & 10:14 & 9:17 & .77 \\
\hline Mean age (years) & $65.5 \pm 10.0$ & $63.2 \pm 10.7$ & .44 \\
\hline Mean body mass index $\left(\mathrm{kg} / \mathrm{m}^{2}\right)$ & $31.2 \pm 6.4$ & $29.2 \pm 6.3$ & .27 \\
\hline Mean CCI scores & $3.0 \pm 0.39$ & $2.5 \pm 0.37$ & .29 \\
\hline Mean hospital length of stay (days) & $3.6 \pm 1.4$ & $3.7 \pm 1.5$ & .25 \\
\hline Active smoker, $n(\%)$ & $4(16.7)$ & $4(15.3)$ & 1.00 \\
\hline
\end{tabular}

Abbreviation: CCI, Charleston Comorbidity Index. ${ }^{30}$

${ }^{1}$ Mean values presented as mean \pm SD. 
Table 3. Patient's surgical characteristics. ${ }^{1}$

\begin{tabular}{lccc}
\hline Variable & Liposomal Bupivacaine Group $(\mathbf{N}=\mathbf{2 4})$ & Control Group (N $=\mathbf{2 6})$ & $\boldsymbol{P}$ Value \\
\hline No. of vertebrae decompressed & $3.5 \pm 0.98$ & $3.7 \pm 0.18$ & .39 \\
No. of vertebrae instrumented & $2.3 \pm 0.5$ & $2.5 \pm 0.6$ & .26 \\
Operation length (min) & $174.4 \pm 59.8$ & $168.7 \pm 56.3$ & .74 \\
Estimated blood loss (mL) & $369.6 \pm 154.1$ & $451.0 \pm 262.5$ & .19 \\
\hline
\end{tabular}

${ }^{1}$ Values presented as mean $\pm \mathrm{SD}$.

preoperatively (5.8 vs. $4.4, P=.13$ ) and postoperatively (5.0 vs. $4.8, P=.80$ ). Likewise, no significant difference in hospital length of stay was found between the treatment and control groups (3.6 vs. 3.7 days, $P=.25$ ).

\section{DISCUSSION}

Most spine surgeries involve deep dissections of subcutaneous tissue, muscle, bone, and ligaments, resulting in postoperative pain that can last for days. $^{7}$ In comparing 179 surgical procedures over a 6-year period, one study reported that single and multilevel spinal fusions were among the top most painful surgeries performed. ${ }^{19}$ It is therefore critical that adequate pain relief be achieved in order to facilitate early mobilization, which has been shown to decrease the hospital length of stay and improve back functionality in patients having undergone spine surgery. ${ }^{20}$ Opioid-based analgesics are known to provide postoperative pain relief but are often associated with serious adverse effects, such as hypotension, altered mental status, respiratory depression, urinary incontinence, and ileus. ${ }^{12}$ Additionally, consuming increased amounts of opioids has been shown to hinder postoperative mobilization and rehabilitation in patients having undergone spine surgery. ${ }^{4,14}$ Current approaches to pain management encourage multimodal regimens in order to reduce opioid consumption and improve postoperative mobilization. ${ }^{4}$ Among them is a nonopioid local anesthetic LB, which has recently been shown to reduce the overall pain, hospital length of stay, and opioid consumption in patients having undergone nonspinal orthopaedic procedures, including total hip and knee arthroplasty. ${ }^{21,22}$ Similar outcomes have also been documented in nonorthopedic cases, including hemorrhoidectomy, bunionectomy, and colorectal and retropubic slingplacement surgeries. ${ }^{16,17,23,24}$ Despite these findings, the local anesthetic's effectiveness in reducing postoperative pain and opioid use in spine surgeries has not been well defined.

This prospective, single-blinded randomized control study was designed to analyze the ability of LB to decrease the total in-hospital opioid requirement following a PLDF for degenerative spondylosis. Although subjects who received intraoperative LB field blocks did have a slightly lower postoperative opioid requirement (normalized to MME) compared to those who received only placebo saline, the difference failed to reach statistical significance. Furthermore, there was no significant difference in opioid consumption among different routes of opioid administration (PCA vs. IV vs. PO) between the treatment and control groups. Moreover, VAS scores between both groups failed to reveal any

Table 4. Postoperative opioid consumption through 72 hours after surgery normalized to morphine milligram equivalent (MME), pre/postoperative visual analog scale scores (VAS), and hospital length of stay between groups. ${ }^{1}$

\begin{tabular}{|c|c|c|c|}
\hline & Liposomal Bupivacaine Group $(\mathrm{N}=\mathbf{2 4})$ & Control Group $(N=26)$ & $P$ Value \\
\hline \multicolumn{4}{|l|}{ Postoperative MME } \\
\hline PCA & $4.5 \pm 3.6$ & $5.1 \pm 4.5$ & .79 \\
\hline $\mathrm{IV}^{2}$ & $1.3 \pm 1.4$ & $1.2 \pm 1.0$ & .83 \\
\hline PO & $5.9 \pm 5.7$ & $7.1 \pm 5.4$ & .35 \\
\hline Total $^{3}$ & $11.6 \pm 9.3$ & $13.4 \pm 8.9$ & .40 \\
\hline \multicolumn{4}{|l|}{ VAS scores } \\
\hline Preoperative & $5.8 \pm 3.2$ & $4.4 \pm 3.3$ & .13 \\
\hline Postoperative day 1 & $5.0 \pm 2.7$ & $5.2 \pm 3.2$ & .46 \\
\hline Postoperative day 2 & $4.8 \pm 2.5$ & $4.7 \pm 2.7$ & .88 \\
\hline Postoperative day 3 & $4.7 \pm 3.3$ & $4.6 \pm 3.1$ & .94 \\
\hline Postoperative days $1-3$ & $5.0 \pm 2.7$ & $4.8 \pm 2.8$ & .80 \\
\hline Hospital length of stay (days) & $3.6 \pm 1.4$ & $3.7 \pm 1.5$ & .25 \\
\hline
\end{tabular}

Abbreviations: PCA, patient-controlled analgesia; IV, intravenous; PO, oral.

${ }^{1}$ Values presented as mean $\pm \mathrm{SD}$.

${ }^{2}$ IV rescue.

${ }^{3}$ Total MME $=$ IV rescue MME + PO MME + PCA MME. 
Table 5. 72-hour postoperative opioid consumption normalized to morphine milligram equivalent (MME) in narcotic-tolerant and narcotic-naive patients. ${ }^{1}$

\begin{tabular}{lccc}
\hline & Liposomal Bupivacaine Group $(\mathbf{N}=\mathbf{2 4})$ & Control Group $(\mathbf{N}=\mathbf{2 6})$ & $\boldsymbol{P}$ Value \\
\hline Narcotic tolerant, n (\%) $^{2}$ & $11(45.8)$ & $9(34.6)$ & .56 \\
Total MME & $13.3 \pm 12.2$ & $20.6 \pm 9.3$ & .048 \\
Narcotic naive, n (\%) & $13(54.1)$ & $17(65.3)$ & .42 \\
Total MME & $10.2 \pm 6.0$ & $9.6 \pm 6.1$ & .72 \\
\hline
\end{tabular}

Abbreviations: IV, intravenous; PO, oral; PCA, patient-controlled analgesia.

${ }^{1}$ Mean values presented with \pm SD.

${ }^{2}$ Total MME $=$ IV rescue MME + PO MME + PCA MME.

significant difference in levels of pain intensity among patients in the postoperative period.

While no difference in postoperative opioid consumption was found between either group, our subanalysis revealed that LB might have a role in reducing the postoperative opioid consumption in NT patients. NT patients who received LB required $35.4 \%$ less total MME than those who received only placebo saline. When comparing NT and NN patients who received saline injections, NT patients required 2.1 times the total MME than NN patients, possibly as a consequence of underlying comorbidities.

The results of this study were similar to those reported by Grieff et al, ${ }^{25}$ who conducted a retrospective chart review of patients undergoing decompression and fusion for cervical or lumbar spondylolisthesis. When compared to bupivacaine HCL, LB was not shown to significantly decrease perioperative opioid use and length of hospitalization. In a similar subanalysis on NT patients, they revealed a trend toward an increased total opioid requirement in the NT subpopulation that received bupivacaine HCL versus LB. The results were similar to the increase in postoperative opioid consumption among NT patients receiving sterile saline versus LB in our study. In 2016, Puffer et $\mathrm{al}^{26}$ compared a prospective cohort of patients who received LB after a single-level lumbar microdiscectomy to a retrospective matched cohort of patients who did not receive local anesthetics. They found that even though patients who received LB collectively spent less time on IV narcotics, this did not translate into a reduction in total MME or VAS pain scores in the postoperative period. However, $\mathrm{Kim}$ et $\mathrm{al}^{27}$ revealed that $\mathrm{LB}$ significantly reduced patient pain scores and opioid consumption only in the first 24 hours following transforaminal lumbar interbody fusion surgery.

Potential benefits of LB in surgical procedures should be weighed against the economic cost to the health care system. LB is an expensive drug with the cost per patient at our institution being $\$ 315.00$. The principal cost benefit of LB would be found in its ability to decrease the hospital length of stay, which our results failed to show. Similarly, Beachler et $\mathrm{al}^{28}$ reported that patients receiving LB during total hip arthroplasty did not have a significant reduction in hospital length of stay and concluded that its use should be scrutinized in our current cost-saving practices.

Several limitations present in our study are worth mentioning. A relatively small sample population was used, and this may obscure statistically significant differences in postoperative opioid consumption between control and treatment groups. In addition, the absence of blinding among research staff and the investigators administering the injections may introduce observer bias. Patients were also given other nonopioid medications postoperatively, and this may affect both primary and secondary outcomes. Although recorded in this study, the presence and severity of comorbidities may also indirectly affect the total postoperative opioid requirement among patients. Finally, one of the secondary outcomes measured in this study was pain intensity, which was assessed using the validated VAS tool. Measuring pain perception is subjective, thus undermining accurate assessments of pain relief in the postoperative period. This could explain a lack of statistical significance of mean VAS scores between the treatment and control groups postoperatively. Despite these limitations, however, our findings provide the highest level of evidence to date on the use of LB in the elective spine surgery population.

\section{CONCLUSIONS}

LB injections did not significantly decrease the postoperative opioid consumption (normalized to MME), pain levels, and hospital length of stay among patients who underwent posterior lumbar decompression and instrumented fusion surgery for degenerative spondylosis compared to patients receiving only saline injections. Our results indicate that the use of LB in patients who are narcotic 
tolerant at the time of surgery may reduce the total postoperative opioid requirement and could possibly be considered as an adjunct to multimodal pain regimens in NT subpopulations undergoing open spine surgery. However, given the study's limitations, no definitive conclusions about the efficacy of LB in spine surgery can be made. Future, welldesigned prospective studies involving large patient populations are needed to clearly assess the efficacy of LB in open spinal procedures.

\section{REFERENCES}

1. Gibson JNA, Waddell G. Surgery for degenerative lumbar spondylosis: updated Cochrane Review. Spine (Phila Pa 1976). 2005;30(20):2312-2320.

2. Zbedlick TA. The treatment of degenerative lumbar disorders: a critical review of the literature. Spine (Phil Pa 1976). 1995; 15(20):126S-137S.

3. Yavin D, Casha S, Wiebe S, et al. Lumbar fusion for degenerative disease: a systematic review and meta-analysis. Neurosurgery. 2017;80(5):701-715.

4. Mathiesen O, Dahl B, Thomsen BA, et al. A comprehensive multimodal pain treatment reduces opioid consumption after multilevel spine surgery. European Spine Journal. 2013;22(9):2089-2096. https://doi.org/10.1007/s00586-0132826-1.

5. Jirarattanaphochai K, Jung S. Nonsteroidal antiinflammatory drugs for postoperative pain management after lumbar spine surgery: a meta-analysis of randomized controlled trials. $J$ Neurosurg Spine. 2008;9(1):22-31.

6. Møiniche S, Mikkelsen S, Wetterslev J, et al. A qualitative systematic review of incisional local anaesthesia for postoperative pain relief after abdominal operations. $\mathrm{Br} J$ Anaes. 1998;81(3):377-383.

7. Bajwa SJS, Haldar R. Pain management following spinal surgeries: an appraisal of the available options. J Craniovertebral Junction Spine. 2015;6(3):105-110.

8. Ziegeler S, Fritsch E, Bauer C, et al. Therapeutic effect of intrathecal morphine after posterior lumbar interbody fusion surgery: a prospective, double-blind, randomized study. Spine (Phila Pa 1976). 2008;33(22):2379-2386.

9. Chan JH, Heilpern GN, Packham I, et al. A prospective randomized double-blind trial of the use of intrathecal fentanyl in patients undergoing lumbar spinal surgery. Spine (Phila Pa 1976). 2006;31(22):2529-2533.

10. Wulf H, Maier C, Schele HA, et al. Plasma concentration of bupivacaine after stellate ganglion blockade. Anesth Analg. 1991;72(4):546-548.

11. Hu D, Onel E, Singla N, et al. Pharmacokinetic profile of liposome bupivacaine injection following a single administration at the surgical site. Clin Drug Investig. 2013;33(2):109-115.

12. Pizzi LT, Toner R, Foley K, et al. Relationship between potential opioid-related adverse effects and hospital length of stay in patients receiving opioids after orthopedic surgery. Pharmacotherapy. 2012;32(6):502-514.

13. American Society of Anesthesiologists Task Force on Acute Pain Management. Practice guidelines for acute pain management in the perioperative setting: an updated report by the American Society of Anesthesiologists Task Force on Acute Pain Management. Anesthesiology. 2004;100:1573-1581.

14. Oderda GM, Said Q, Evans RS, et al. Opioid-related adverse drug events in surgical hospitalizations: impact on costs and length of stay. Ann Pharmacother. 2007;41(3):400-406.

15. Dasta J, Ramamoorthy S, Patou G, et al. Bupivacaine liposome injectable suspension compared with bupivacaine HCL for the reduction of opioid burden in the postsurgical setting. Curr Med Res Opin. 2012;28(10):1609-1615.

16. Golf M, Daniels SE, Onel E. A phase 3 randomized placebo controlled trial of Depofoam bupivacaine (extended release bupivacaine local analgesic) in bunionectomy. Adv Ther. 2011;28(9):776-788.

17. Gorfine SR, Onel E, Patou G, et al. Bupivacaine extended-release liposome injection for prolonged postsurgical analgesia in patients undergoing hemorrhoidectomy: a multicenter, randomized, double-blind, placebo-controlled trial. Dis Colon Rectum. 2011;54(12):1552-1559.

18. Smoot JD, Bergese SD, Onel E, et al. The efficacy and safety of DepoFoam bupivacaine in patients undergoing bilateral, cosmetic, submuscular augmentation mammoplasty: a randomized, double-blind, active-control study. Aesthet Surg J. 2012;32(1):69-76.

19. Gerbershagen HJ, Aduckathil S, van Wijck AJ, et al. Pain intensity on the first day after surgery: a prospective cohort study comparing 179 surgical procedures. Anesthesiology. 2013;118(4):934-944.

20. Epstein NE. A review article on the benefits of early mobilization following spinal surgery and other medical/ surgical procedures. Surg Neurol Int. 2014;5(suppl 3):S66-S73.

21. Bramlett K, Onel E, Viscusi ER, et al. A randomized, double-blind, dose-ranging study comparing would infiltration of DepoFoam bupivacaine, an extended-release liposomal bupivacaine, to bupivacaine HCL for postsurgical analgesia in total knee arthroplasty. The Knee. 2012;19(5):530-536.

22. McGraw-Tatum MA, Groover MT, George NE, et al. A prospective, randomized trial comparing liposomal bupivacaine vs fascia iliaca compartment block for postoperative pain control in total hip arthroplasty. $J$ Arthroplasty. 2017;32(7):2181-2185.

23. Stokes AL, Adhikary SD, Quintili A, et al. Liposomal bupivacaine use in transversus abdominis plane blocks reduces pain and postoperative intravenous opioid requirement after colorectal surgery. Dis Colon Rectum. 2017;60(2):170-177.

24. Mazloomdoost D, Pauls RN, Hennen EN, et al. Liposomal bupivacaine decreases pain following retropubic sling placement: a randomized placebo-controlled trial. $\mathrm{Am} \mathrm{J}$ Obstet Gynecol. 2017;217(5):598.e1-598.e11.

25. Grieff AN, Ghobrial GM, Jallo J. Use of liposomal bupivacaine in the postoperative management of posterior spinal decompression. J Neurosurg Spine. 2016;25(1):88-93.

26. Puffer RC, Tou K, Winkel RE, et al. Liposomal bupivacaine incisional injection in single-level lumbar spine surgery. Spine J. 2016;16(11):1305-1308.

27. Kim J, Burke SM, Kryzanski JT, et al. The role of liposomal bupivacaine in reduction of postoperative pain after transforaminal lumbar interbody fusion: a clinical study. Word Neurosurg. 2016;91:460-467.

28. Beachler JA, Kopolovich DM, Tubb CC, et al. Liposomal bupivacaine in total hip arthroplasty: do the results justify the cost? J. Orthop. 2017;14(1):161-165. 
29. Saraghi M, Hersch EV. Three newly approved analgesics: an update. Anesth Prog. 2013;60(4):178-187.

30. Charlson ME, Pompei P, Ales KL, et al. A new method of classifying prognostic comorbidity in longitudinal studies: development and validation. J Chronic Dis. 1987;40(5):373-383.

Disclosures and COI: $\mathrm{LB} / \mathrm{TW} / \mathrm{MS} / \mathrm{OY} / \mathrm{IY} /$ OT: No disclosures. SL: American Board of Orthopaedic Surgery, Inc Board or committee member, American Orthopaedic Association: Board or committee member. AO Spine North America Spine Fellowship Support: Research support. ASIP, ISD: Stock or stock Options. Cervical Spine Research Society: Board or committee member. DePuy, A Johnson \& Johnson Company: IP royalties; Paid consultant; Paid presenter or speaker. Globus Medical: Paid consultant; Research support. Journal of Spinal Disorders and Techniques: Editorial or governing board. K2M spine: Research support. K2Medical: Paid consultant. OMEGA: Research support. Pacira: Research support. SMISS: Board or committee member. Synthes: Paid consultant; Paid presenter or speaker. Thieme, QMP: Publishing royalties, financial or material support. DG:
Advanced Spinal Intellectual Property: Stock or stock options. Depuy-Synthes Spine: IP royalties; Paid presenter or speaker. Globus Medical: IP royalties. EK: Biomet: Paid consultant. DePuy, A Johnson \& Johnson Company: Paid presenter or speaker. KB: K2M: Employee. Orthofix, Inc: Research support. Institutional review board approval was obtained before conducting this research study. Funding: This study was funded by Pacira Pharmaceutical, Inc.

Corresponding Author: Steven Ludwig, MD, Department of Orthopaedics, University of Maryland Medical Center, 110 South Paca Street, 6th Floor, Suite 300, Baltimore, MD 21201. Phone: (410) 328-3330; Fax: (410) 328-0534; Email: sludwig@umoa.umm.edu.

Published 31 August 2018

This manuscript is generously published free of charge by ISASS, the International Society for the Advancement of Spine Surgery. Copyright (C) 2018 ISASS. To see more or order reprints or permissions, see http://ijssurgery.com. 